DOI: 10.12957/demetra.2017.26201

\title{
Presión arterial en niños y adolescentes de Montevideo según el patrón de distribución de la grasa corporal
}

\section{Blood pressure and pattern of body fat distribution in children and adolescents of Montevideo}

\author{
Estela Skapino \\ María Isabel Bove ${ }^{2}$ \\ Raúl Ramírez ${ }^{3}$ \\ 1 Universidad de la República, Escuela de \\ Nutrición. Montevideo, Uruguay. \\ ${ }^{2}$ Universidad Católica del Uruguay, Instituto de \\ Bienestar y Salud. Montevideo, Uruguay. \\ ${ }^{3}$ Universidad de la República, Departamento \\ de Métodos Cuantitativos, FCEyA. Montevideo, \\ Uruguay. \\ Correspondencia / Correspondence \\ María Isabel Bove \\ isabelbove.uru@gmail.com
}

\section{Resumen}

Introducción: La hipertensión a edades tempranas es determinante para la evolución negativa de las enfermedades cardiovasculares en la vida adulta. Objetivo: Relacionar los niveles de presión arterial con el patrón de distribución de grasa corporal en un grupo de niños y adolescentes de Montevideo. Metodología: Se tomó una muestra por conveniencia de 47 preescolares, 44 escolares y 41 adolescentes. Se midió su peso, estatura, circunferencia de cintura y brazo y pliegues subcutáneos, que permitieron construir indicadores de patrón de distribución de grasa corporal, y también su presión arterial sistólica y diastólica. Las correlaciones se estudiaron a través del coeficiente de correlación lineal de Pearson. Para las variables que podrían influir en la presión arterial, se realizó un modelo de regresión lineal. Resultados: Se observó una presión arterial alterada en el 9,8\% de la población estudiada, sin diferencias significativas entre los sexos ni relacionadas a la edad. El exceso de grasa central se observó en $32,7 \%$, y el exceso de grasa periférica en $18,2 \%$, sin diferencias estadísticamente significativas por sexo. La circunferencia de cintura mostró una correlación positiva moderada con la presión sistólica $(\mathrm{R}=0,618)$, y una relación débil con el área grasa del brazo $(\mathrm{R}=0,414)$. Según el modelo de regresión lineal, por cada centímetro de cintura que aumenta, se espera que la presión sistólica aumente $0,619 \mathrm{mmHg}$. Conclusión: a través del presente trabajo se observó un patrón de distribución de grasa corporal centrípeta, sin diferencias según sexo, moderadamente relacionado con la presión arterial, por lo que es necesario incorporar en la práctica pediátrica el uso de la circunferencia de cintura y el control de la presión arterial como predictores de riesgo metabólico. 
Palabras clave: Presión Arterial, Niños y Adolescentes, Distribución de Grasa Corporal.

\section{Abstract}

Introduction: Hypertension (HTA) at an early age is determinative of the negative development of cardiovascular disease in adulthood. Objective: To relate the levels of blood pressure with the pattern of body fat distribution in a group of children and adolescents of Montevideo. Methodology: We worked with a convenient sample of 47 preschool children, 44 school children and 41 adolescents. We measured their weight, height, waist and arm circumferences and subcutaneous folds. These measures allowed the construction of patterns of body fat distribution indicators. We measured their systolic and diastolic blood pressure. Correlations were studied through Pearson linear correlation coefficient. For the variables that might influence blood pressure, a linear regression model was performed. Results: Blood pressure was altered in $9.8 \%$ of the study population, with no significant differences by gender or age. Excess of central fat was observed in $32.7 \%$ of the population and excess of peripheral fat in $18.2 \%$, with no statistically significant differences by gender. The waist circumference showed a moderate positive correlation with systolic pressure $(\mathrm{R}=0.618)$, and a weak relationship with the arm fat area $(R=0.414)$. According to the linear regression model, for each centimeter of waist increment, systolic blood pressure is expected to increase $0.619 \mathrm{mmHg}$. Conclusions: Through this study we observed a centripetal body fat distribution pattern with no differences by gender and moderately related to PA. Therefore it is necessary to incorporate in the pediatric practice the use of waist circumference and the control of blood pressure as predictors of metabolic risk.

Keywords: Blood Pressure, Children and Adolescents, Body Fat Distribution.

\section{Introducción}

Las enfermedades relacionadas con los estilos de vida, especialmente las enfermedades cardiovasculares (ECV), son la principal causa de muerte en el mundo ${ }^{1,2}$, y la hipertensión arterial (HTA) es uno de los factores de riesgo más importantes para el desarrollo de esas enfermedades. La presencia de HTA en la infancia se vincula con su presencia en la edad adulta. ${ }^{3}$ Se ha observado 
que los niños que se encuentran en los niveles superiores en la distribución de la presión arterial tienden a presentar los mismos niveles en la distribución de presión arterial en la edad adulta. ${ }^{4}$

Aunque los estudios sobre la prevalencia de presión arterial (PA) elevada en la población pediátrica son relativamente escasos, se ha visto un aumento de su prevalencia en los últimos años, ${ }^{5,6}$ por lo que ha sido motivo de varios consensos. ${ }^{7,8}$ El avance de la HTA en edad temprana se ha relacionado al paralelo incremento de la obesidad, tal como se ha demostrado en países como Estados Unidos ${ }^{9}$, Venezuela ${ }^{10}$ y Méjico. ${ }^{11}$ Además, los resultados de estudios epidemiológicos con seguimiento a medio o largo plazo corroboran que la HTA a edades tempranas es determinante para la evolución negativa de enfermedades cardiovasculares en la vida adulta. ${ }^{2-14}$ De tal evidencia, se desprende la importancia de detectar la HTA infantil antes de que sus complicaciones ocasionen problemas de salud en etapas posteriores de la vida.

La obesidad se define por un aumento de la grasa corporal. ${ }^{15}$ Esta puede estar distribuida de manera uniforme en todo el cuerpo o predominar fundamentalmente en la región abdominal. ${ }^{16}$ El IMC es un indicador que se utiliza para definir la masa corporal total y que insinúa, en caso de estar elevado, que existe un depósito graso excesivo. La confirmación de que un valor elevado de IMC se debe a un exceso de grasa se puede realizar a través de la medición del tejido subcutáneo, ya que aproximadamente la mitad de la grasa del cuerpo se deposita debajo de la piel, o de la circunferencia de la cintura, que revela el depósito graso a nivel visceral. ${ }^{17}$ La grasa abdominal o visceral, que sirve primordialmente para la movilización fácil y rápida de reservas energéticas, está estrechamente relacionada con un aumento de la prevalencia de enfermedades metabólicas, incluyendo la enfermedad cardiovascular.

El presente estudio tiene como objetivo relacionar los niveles de presión arterial con el patrón de distribución de grasa corporal en un grupo de individuos preescolares, escolares y adolescentes de Montevideo.

\section{Metodología}

Se trata de un estudio epidemiológico observacional de corte transversal. Se trabajó con una muestra por conveniencia compuesta por 47 niños en edad preescolar, 44 en edad escolar y 41 adolescentes que concurrían al Jardín de Infantes $N^{\circ} 362$, Escuela $N^{\circ} 122$ y Centro Espigas, instituciones de educación pública de la ciudad de Montevideo, entre los meses de abril y mayo de 2016. Se excluyeron las adolescentes embarazadas.

Los directores de los centros educativos seleccionados recibieron una solicitud formal y detallada sobre la importancia, los objetivos y la metodología del estudio, por medio de la cual pudieron dar su consentimiento para colaborar con el proyecto de forma voluntaria. 
Este estudio fue aprobado por el Comité de Ética e Investigación de la Universidad Católica del Uruguay. Se coordinaron reuniones con los padres de los niños preescolares y escolares, así como también con los adolescentes, con el fin de explicarles el motivo de la investigación y solicitarles el consentimiento para su realización.

En un segundo momento, se concurrió a realizar las mediciones antropométricas de los participantes. Las mediciones fueron hechas por un grupo de nutricionistas entrenado, utilizando técnicas debidamente estandarizadas. ${ }^{18,19}$ Los participantes fueron pesados y medidos en ropas livianas y descalzos. El peso se tomó en una balanza digital Seca ${ }^{\circledR}$ modelo 841 (100g de precisión). La talla se midió en tallímetro de pie Seca ${ }^{\boxplus}$ (0,1 $\mathrm{cm}$ de precisión). Se calculó el índice de masa corporal $\left(\mathrm{IMC}=\mathrm{Peso} / \mathrm{Talla}^{2}\right)$. Se midió la circunferencia braquial con cinta métrica inextensible marca Seca ${ }^{\circledR}$ y el pliegue tricipital se tomó utilizando un cáliper Lange ${ }^{\circledR}$.

La medida de la circunferencia de cintura se realizó de acuerdo a lo establecido por la OMS, en el plano que pasa por el punto medio entre el reborde costal inferior y la cresta ilíaca, utilizando la cinta métrica ya mencionada. Cada medición se realizó por duplicado en forma no consecutiva. El indicador IMC/Edad se comparó con las tablas de referencia de la OMS. ${ }^{20} \mathrm{El}$ área de grasa braquial (AGB) se determinó a través de las fórmulas de Frisancho y se comparó con la tabla de referencia del mismo autor. ${ }^{21}$ Los valores de circunferencia de cintura (CC) se compararon con las tablas del NHANES III ${ }^{22}$ y se los consideró CC aumentados cuando se encontraban por encima del percentil 90 .

La PA se midió con un monitor de presión arterial automático de la marca OMRON, previamente calibrado con un esfigmomanómetro de mercurio. Se realizaron dos tomas separadas por cinco minutos. Se cotejaron las diferencias en los resultados y, en los casos en que se encontraron diferencias mayores al 5\% en alguna de las dos presiones (sistólica y/o diastólica), se procedió a una tercera medición de la PA. Las medidas de PA se compararon con las establecidas en el 3er Consenso Uruguayo de Hipertensión Arterial en el Niño y el Adolescente. ${ }^{8}$ Se consideró la presión arterial alterada cuando la presión arterial sistólica (PAS) y/o diastólica (PAD) se encontraba por encima del percentil 95 según el sexo y la edad.

Análisis estadístico: Las variables cuantitativas se expresaron a través de los valores de media con un intervalo de confianza al 95\%, y las variables cualitativas a través de la frecuencia absoluta y porcentajes. Las variables fueron analizadas a través de la prueba de Student para muestras independientes y de la prueba de hipótesis de $\mathrm{Chi}^{2}$, respectivamente. Las correlaciones se estudiaron a través de la $\mathrm{R}$ de Pearson ( $\mathrm{R}$ al cuadrado corregida) y del Modelo de Regresión lineal.

Todas las pruebas se consideraron estadísticamente significativas con un nivel $(\alpha)$ de 0.05 . Para el procesamiento de los datos, se utilizó el programa IBM SPSS, Statistics 20. 


\section{Resultados}

Se relevaron datos de 132 niños y adolescentes: 35,6\% en edad preescolar ( $n=47), 33,3 \%$ en edad escolar $(n=44)$ y $31,1 \%(n=41)$ adolescentes. La distribución por sexo fue pareja en cada uno de los rangos de edad.

En la Tabla 1, se pueden observar los valores de media e intervalo de confianza del $95 \%$ de las variables antropométricas estudiadas diferenciadas por sexo. Los resultados indican un perfil morfológico similar entre niños y niñas y presentan alguna diferencia sólo en el área de grasa medida a través del pliegue trícipital $(\mathrm{p}=0,001)$ y del área de grasa $(\mathrm{p}=0,008)$, en donde las niñas presentaron valores ligeramente superiores a los de los niños.

Tabla 1. Media e intervalo de confianza del 95\% de las variables antropométricas estudiadas por sexo.

\begin{tabular}{lllll}
\hline & Varones & Mujeres & Total & $\mathrm{p}$ \\
\hline Peso $(\mathrm{Kg})$. & $37,5(32,3-42,7)$ & $37,4(32,5-42,2)$ & $37,4(33,8-41,1)$ & NS \\
Estatura $(\mathrm{cm})$ & $132,2(125,6-138,7)$ & $134,1(127,2-140,9)$ & $132,9(128,2-137,7)$ & NS \\
IMC $\left(\mathrm{Kg} / \mathrm{m}^{2}\right)$ & $19,1(18,2-20,1)$ & $19,5(18,6-20,5)$ & $19,3(18,6-19,9)$ & NS \\
Circunferencia del brazo $(\mathrm{mm})$ & $206,6(194,4-218,7)$ & $216,9(203,4-230,4)$ & $210,6(201,6-219,6)$ & NS \\
Pliegue tríceps $(\mathrm{mm})$ & $10,7(9,6-11,8)$ & $14,0(12,4-15,6)$ & $12,0(11,1-12,9)$ & 0,001 \\
Área de grasa $\left(\mathrm{mm}^{2}\right)$ & $1065(913-1217)$ & $1422(1195-1650)$ & $1205(1074-1335)$ & 0,008 \\
Área muscular $\left(\mathrm{mm}^{2}\right)$ & $2525(2199-2852)$ & $2470(2154-2785)$ & $2504(2273-2735)$ & NS \\
Circunferencia de cintura $(\mathrm{cm})$ & $60,9(57,8-64,0)$ & $62,9(59,7-66,1)$ & $61,7(59,4-63,9)$ & NS \\
\hline
\end{tabular}

Se observó una presión arterial alterada (>P95 según edad y sexo) en el 9,8\% en la población estudiada. La alteración de la PAS se verificó en el 6,8\% de la muestra y de la PAD en 6,1\%. No se encontraron diferencias significativas entre varones y mujeres, así como tampoco entre los rangos de edad (Tabla 2) 
Tabla 2. Hipertensión arterial y exceso de grasa corporal.

\begin{tabular}{lllll}
\hline & Varones & Mujeres & Total & $\mathrm{p}$ \\
\hline $\mathrm{N}$ & 79 & 53 & 132 & \\
Hipertensión según la edad & & & & \\
Sistólica > P95 & $8,9 \%$ & $3,8 \%$ & $6,8 \%$ & $\mathrm{NS}$ \\
Diastólica > P95 & $6,3 \%$ & $5,7 \%$ & $6,1 \%$ & $\mathrm{NS}$ \\
Sistólica o diastólica > P95 & $12,7 \%$ & $5,7 \%$ & $9,8 \%$ & $\mathrm{NS}$ \\
Exceso de grasa según la localización y edad & & & & \\
Central (Circunferencia de Cintura > P75) & $32,8 \%$ & $32,6 \%$ & $32,7 \%$ & $\mathrm{NS}$ \\
Central (Circunferencia de Cintura > P90) & $9,0 \%$ & $11,6 \%$ & $10,0 \%$ & $\mathrm{NS}$ \\
Periférica (Área de Grasa del Brazo > P75) & $26,8 \%$ & $37,2 \%$ & $30,9 \%$ & $\mathrm{NS}$ \\
Periférica (Área de Grasa del Brazo > P90) & $16,4 \%$ & $20,9 \%$ & $18,2 \%$ & $\mathrm{NS}$ \\
\hline
\end{tabular}

Por otra parte, el exceso de grasa central estimado a través de circunferencia de cintura > P75 se observó en el 32,7\%, el exceso de grasa periférica estimado a través del área grasa del brazo > P90 en el 18,2\% y no se observaron diferencias estadísticamente significativas por sexo (Tabla 2).

$\mathrm{Al}$ analizar las correlaciones de la PAS y PAD con la circunferencia de la cintura y con el área de grasa del brazo, se observó una significación positiva para ambas. Se puede observar que la grasa central, expresada por la circunferencia de la cintura, muestra una correlación positiva moderada con la presión sistólica $(\mathrm{R}=0,618)$ y que la grasa periférica, expresada a través del área de grasa del brazo ( $\mathrm{R}=0,414)$, muestra una relación débil con esa misma presión (Figura 1). 

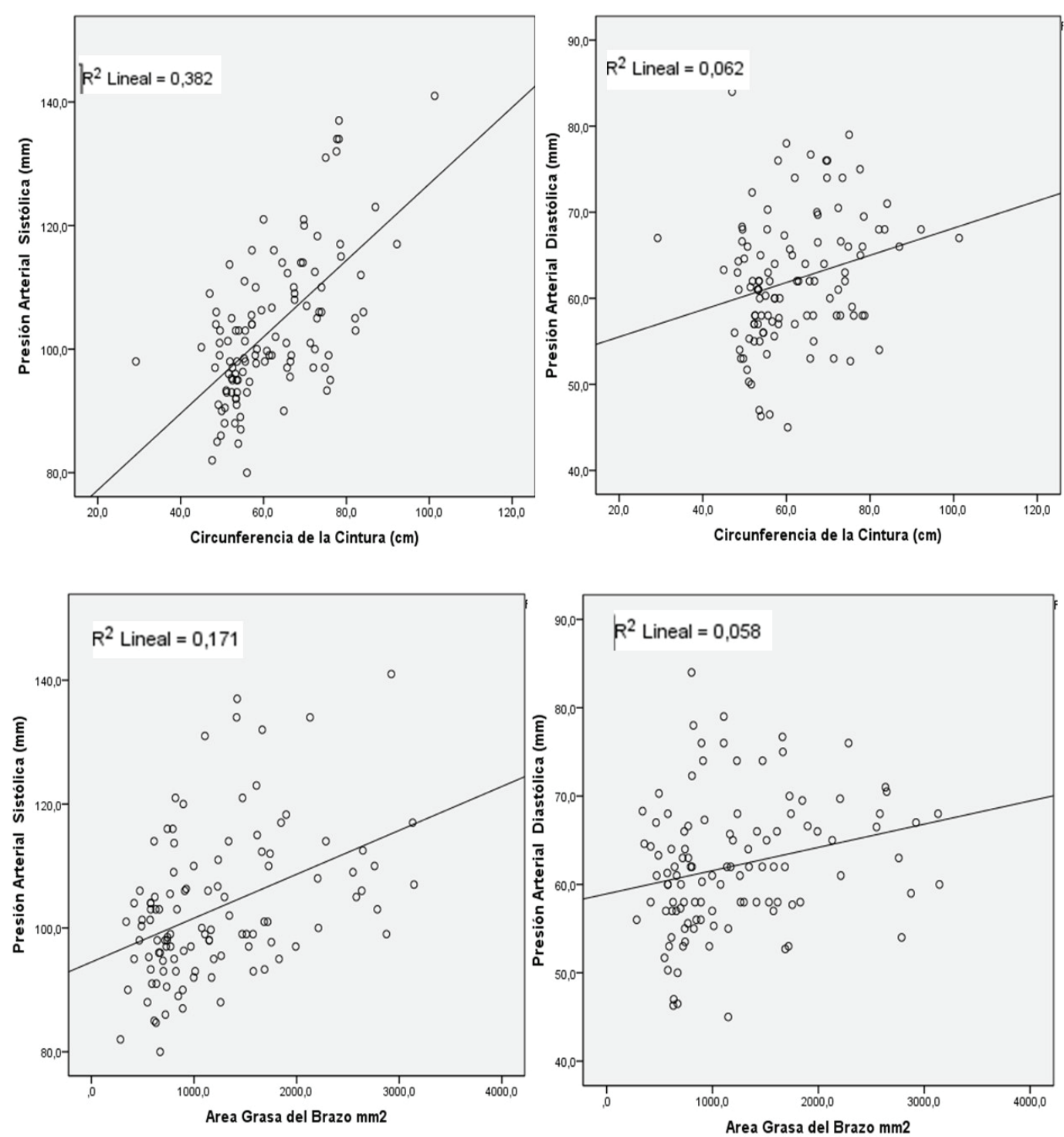

Figura 1. Relación de la Presión Arterial Diastólica y Sistólica con la Circunferencia de la Cintura y el Área de Grasa del Brazo. 
Según el modelo de regresión lineal, por cada centímetro de cintura que se aumenta, se espera que la presión arterial sistólica aumente 0,619 mmHg (Tabla 3)

Tabla 3. Modelo de regresión lineal de la Presión Arterial Sistólica y Diastólica en relación a la circunferencia de la cintura y al Área de Grasa del Brazo

\begin{tabular}{|c|c|c|c|c|c|c|c|}
\hline Modelo de PAS & $\mathrm{R}$ & $\begin{array}{l}\mathrm{R} \\
\text { cuadrado }\end{array}$ & $\begin{array}{l}\mathrm{R} \text { cuadrado } \\
\text { corregida }\end{array}$ & B & $\begin{array}{l}\text { Límite } \\
\text { inferior }\end{array}$ & $\begin{array}{l}\text { Límite } \\
\text { superior }\end{array}$ & Sig. \\
\hline $\begin{array}{l}\text { PAS- Circunferencia } \\
\text { de la cintura }\end{array}$ & 0,618 & 0,382 & 0,376 & 0,619 & 0,469 & 0,77 & $<0,001$ \\
\hline (Constante) & & & & 64,82 & 55,38 & 74,257 & $<0,001$ \\
\hline $\begin{array}{l}\text { PAS- Área de Grasa } \\
\text { del Brazo }\end{array}$ & 0,414 & 0,171 & 0,163 & 0,007 & 0,004 & 0,01 & $<0,001$ \\
\hline (Constante) & & & & 94,49 & 90,362 & 98,621 & $<0,001$ \\
\hline \multicolumn{8}{|l|}{ Modelo de PAD } \\
\hline $\begin{array}{l}\text { PAD- Circunferencia } \\
\text { de la cintura }\end{array}$ & 0,248 & 0,062 & 0,053 & 0,158 & 0,04 & 0,275 & 0,009 \\
\hline (Constante) & & & & 52,36 & 44,981 & 59,742 & $<0,001$ \\
\hline $\begin{array}{l}\text { PAD- Área de Grasa } \\
\text { del Brazo }\end{array}$ & 0,242 & 0,058 & 0,05 & 0,003 & 0,001 & 0,005 & 0,011 \\
\hline (Constante) & & & & 58,94 & 56,145 & 61,73 & $<0,001$ \\
\hline
\end{tabular}

\section{Discusión}

El presente estudio se propuso relacionar los niveles de presión arterial con el patrón de distribución de grasa corporal en un grupo de individuos preescolares, escolares y adolescentes de Montevideo. Si bien el trabajo presenta sus limitaciones, ya que se trata de una muestra por conveniencia, de corte transversal y sin repetición de la toma de la presión arterial en días sucesivos, tiene la fortaleza de determinar la grasa subcutánea a través de la medición del pliegue trícipital, algo poco documentado en nuestro medio. 
La presión arterial elevada en la infancia es una manifestación temprana de la hipertensión en la edad adulta. Son muchos los factores que pueden ser determinantes en el desarrollo de esa patología en la niñez: la presión arterial materna durante el embarazo, el bajo peso al nacer, la posición socioeconómica adversa, el elevado consumo de sodio y la adiposidad en la infancia. ${ }^{23-25}$ Es en este último aspecto que el presente trabajo se ha centrado al medir la grasa abdominal y subcutánea y observar su correlación con la presión arterial.

Al igual que lo observado por otros autores, las mejores correlaciones verificadas en el presente estudio fueron las correspondientes a la circunferencia de la cintura con la presión sistólica. La grasa subcutánea mostró menor correlación con la presión arterial que la grasa depositada en el del abdomen. Por otra parte, la medición de los pliegues cutáneos, además de mostrar una sensibilidad menor que el de la circunferencia de la cintura, resulta difícil y requiere personal especializado. La circunferencia de la cintura refleja la grasa abdominal con mayor sensibilidad y especificidad que otros indicadores como la relación cintura/cadera, cintura/ talla e IMC ${ }^{26}$ y es de gran utilidad tanto clínica como epidemiológica.

La circunferencia de la cintura en los niños y adolescentes es un buen predictor de las complicaciones metabólicas y de riesgo cardiovascular. ${ }^{27,28}$ El Bogalusa Heart Study ${ }^{29}$ demostró que la distribución de la grasa central determinada por la circunferencia de la cintura en edades entre 5 y 17 años, como las estudiadas en el presente trabajo, está asociada con concentraciones anormales de triglicéridos, LDL-C, HDL-C e insulina, es decir, que la obesidad central en comparación con la obesidad generalizada está más fuertemente correlacionada con los factores de riesgo metabólicos ${ }^{30}$, por lo que la obesidad central es un factor de riesgo independiente para el desarrollo de diabetes tipo 2 , dislipidemia y enfermedad coronaria. ${ }^{31}$

Dado que el patrón de distribución de la grasa corporal se perfila desde etapas tempranas de la vida y que predispone a padecer en el futuro de enfermedades no transmisibles, es necesaria la prevención, la detección precoz de la población en riesgo y la implementación de medidas de atención. Tomando en cuenta la situación epidemiológica nutricional de los niños en nuestro país, se recomienda el uso de indicadores de patrón de distribución de grasa corporal para identificar precozmente la población pediátrica en mayor riesgo de enfermedades no transmisibles.

\section{Conclusiones}

A través del presente trabajo se observó un patrón de distribución de grasa corporal centrípeta, sin diferencias según el sexo moderadamente relacionadas con la PA, por lo que es necesario incorporar en la práctica pediátrica el uso de la medición de la circunferencia de cintura y el control de la PA como predictores de riesgo metabólico. 


\section{Agradecimientos}

Se agradece a las familias, niños y adolescentes que accedieron a participar en el presente proyecto y a Nestlé Uruguay por su financiamiento.

\section{Participación}

Skapino E: Trabajo de campo, participación en la redacción del artículo a publicar; Bove MI: Diseño de la investigación, interpretación y discusión de los resultados en el artículo a publicar; Ramirez R: Procesamiento de los datos, realización de pruebas estadísticas, diseño de gráficos y tablas a publicar.

Conflicto de Intereses: Los autores declaran no haber conflicto de intereses.

\section{Referencias}

1. Wang Y, Monteiro C, Popkin BM. Trends of obesity and underweight in older children and adolescents in the United States, Brazil, China, and Russia. Am J Clin Nutr. 2002; 75(6):971-7.

2. Kelishadi R. Childhood overweight, obesity, and the metabolic syndrome in developing countries. Epidemiol Rev. 2007; 29:62-76

3. Symonds ME, Stephenson T, Budge H. Early determinants of cardiovascular disease: the role of early diet in later blood pressure control. Am J Clin Nutr. 2009; 89(5):1518S-1522S.

4. Lo K, Wong M, Khalechelvam P, Tam W. Waist-to-height ratio, body mass index and waist circumference for screening paediatric cardio-metabolic risk factors: a meta-analysis. Obes Rev. 2016; 17(12)1258-1275.

5. Gambetta JC, Haladjian M, Castillos J, Seré G, Blanco C, Sayaguez B, et al. Obesidad y factores de riesgo cardiovascular en la edad pediátrica. Arch Pediatr Urug. 2008; 79:7-14.

6. Sandoya E, Puppo T, Vázquez H, Portos A, Castro M, Fort Z. Evolución de la hipertensión arterial en Uruguay: 1948-2011. Rev Urug Cardiol. 2012; 27(3):377-386.

7. Gazzara G, coordenador. Consenso de hipertensión arterial en el niño y el adolescente. Arch Pediatr Urug. 2002; 73(1):26-31

8. Caggiani M, Farré Y, coordenadores. 3er Consenso Uruguayo de Hipertensión Arterial en el Niño y el Adolescente. Arch Pediatr Urug. 2006; 77(3):300-305

9. Meininger JC, Brosnan CA, Eissa MA, Nguyen TQ, Reyes LR, Upchurch SL, et al. Overweight and central adiposity in school-age children and links with hypertension. J Pediatr Nurs. 2010; 25:119-25.

10. Blanco-Cedres L, Macias-Tomei C, López-Blanco M. Relación entre la maduración temprana, índice de masa corporal y el comportamiento longitudinal de la presión arterial sistólica. Acta Cient Venez. 2000; 51(4):252-256. 
11. Bojórquez Díaz CI, Angulo CM, Reynoso Erazo L. Factores de riesgo de hipertensión arterial en niños de primaria. Psicología y Salud 2011; 21:245-52

12. Chen X, Wang Y. Tracking of blood pressure from childhood to adulthood: a systematic review and meta-regression analysis. Circulation 2008; 117:3171-80.

13. Virdis A, Ghiadoni L, Masi S, Versari D, DaghiniE, Giannarelli C, et al. Obesity in the childhood: a link to adult hypertension. Curr Pharm Des. 2009; 15:1063-71.

14. Srikanthan K, Feyh A, Visweshwar H, Shapiro JI, Sodhi K. Systematic review of metabolic syndrome biomarkers: a panel for early detection, management, and risk stratification in the west virginian population. Int J Med Sci. 2016; 13(1):25-38.

15. Daza CH. La obesidad: un desorden metabólico de alto riesgo para la salud. Colomb Med. 2002; 33(2):72-80.

16. Okada R, Yasuda Y, Tsushita K, Wakai K, Hamajima N, Matsuo S. Upper-normal waist circumference is a risk marker for metabolic syndrome in normal-weight subjects. Nutrition, Metabolism \& Cardiovascular Diseases 2016; 26(1):67-76.

17. Ritchie SA, Connell JM. The link between abdominal obesity, metabolic syndrome and cardiovascular disease. Nutr Metab Cardiovasc Dis. 2007; 17(4):319-26.

18. Durnim JVGA, Rahaman MM. The assessment of the amount of fat in the human body from measurements of skinfold thickness. Br J Nutr. 1967; 21:681-8.

19. Martin AD, Drinkwater DT. Variability in the measures of body fat. Assumptions or techniques? Sports Med. 1991; 11:277-88

20. Organización Mundial de la Salud. Patrones de crecimiento infantile. Disponível em: www.who.int/ childgrowth/standards/imc_para_edad/es/

21. Frisancho AR. New norms of upper limb fat and muscle areas for assessment of nutritional status. Am J Clin Nutr. 1981; 34(11):2540-5.

22. Mzayek F, Cruickshank JK, Amoah D, Srinivasan S, Chen W, Berenson GS. Birth weight was longitudinally associated with cardiometabolic risk markers in mid-adulthood. Ann Epidemiol. 2016; 26(9):643-7.

23. Tanvig M. Offspring body size and metabolic profile - effects of lifestyle intervention in obese pregnant women. Dan Med J. 2014; 61(7):B4893.

24. Tanvig M, Vinter CA, Jørgensen JS, Wehberg S, Ovesen PG, Beck-Nielsen H, et al. Effects of lifestyle intervention in pregnancy and anthropometrics at birth on offspring metabolic profile at 2.8 years: results from the Lifestyle in Pregnancy and Offspring (LiPO) study. J Clin Endocrinol Metab. 2015; 100(1):175-83.

25. Freedman DS, Serdula MK, Srinivasan SR, Berenson GS. Relation of circumferences and skinfold thicknesses to lipid and insulin concentrations in children and adolescents: the Bogalusa Heart Study. Am J Clin Nutr. 1999; 69:308-317. 
26. Mc Carthy HD, Ellis SM, Cole TJ. Central overweight and obesity in British youth aged 11-16 years: cross sectional surveys of waist circumference. BMJ 2003; 326:624-632

27. Piazza N. La circunferencia de cintura en los niños y adolescentes Arch Argent Pediatr. 2005; 103(1):5-6.

28. Zhang T, Zhang H, Li Y, Sun D, Li S, Fernandez C, et al. Temporal relationship between childhood body mass index and insulin and its impact on adult hypertension: The Bogalusa Heart Study. Hypertension 2016; 68(3):818-23.

29. Zhang H, Zhang T, Li S, Li Y, Hussain A, Fernandez C, et al. Long-term impact of childhood adiposity on adult metabolic syndrome is modified by insulin resistance. The Bogalusa Heart Study. Sci Rep. 2015; 5:17885.

30. Maffeis C, Pietrobelli A, Grezzani A, Provera S, Tato L. Waist circumference and cardiovascular risk factors in prepubertal children. Obes Res. 2001; 9:179-187.

31. Kelishadi R, Mirmoghtadaee P, Najafi H, Keikha M. Systematic review on the association of abdominal obesity in children and adolescents with cardio-metabolic risk factors. J Res Med Sc. 2015; 20(3):294-307. 